\title{
Nonlinearity Effects of Lateral Density Diffusion Coefficient on Gain-guided VCSEL Performance
}

\author{
Jianzhong Li, Samson H. Cheung, and C. Z. Ning \\ Center for Nanotechnology, \\ NASA Ames Research Center, M/S T27A-1, Moffett Field, CA94035, U.S.A.
}

\begin{abstract}
Electron and hole diffusions in the plane of semiconductor quantum wells play an important part in the static and dynamic operations of semiconductor lasers. In this paper, we apply a hydrodynamic model developed from the semiconductor Bloch equations to numerically study the effects of nonlinearity in the diffusion coefficient on single mode operation and direct modulation of a gain-guided InGaAs/GaAs multiple quantum well laser, operating not too far from threshold. We found that a small diffusion coefficient is advantageous for lowering the threshold current and increasing the modulation bandwidth. Most importantly, the effects of nonlinearity in the coefficient can be approximately reproduced by replacing the coefficient with an effective constant diffusion coefficient, which corresponds roughly to the half height density of the density distribution. This conclusion is the same as in Ref. 8, but we will discuss the disagreements in reaching it. Finally, the beam profile is slightly modified in the nonlinear case.
\end{abstract}

Keywords: semiconductor laser, inhomogeneity, lateral carrier diffusion, nonlinearity, VCSEL

\section{INTRODUCTION}

Non-uniformities in semiconductor lasers are known to influence the operating parameters of the devices, ${ }^{1}$ such as the threshold current. Extrinsic causes, such as interface roughness along the quantum well (QW) and doping profile in the QW structure, could result in such inhomogeneities, and surely could intrinsic reasons. For instance, carrier concentration distribution and the associated diffusion in the lateral active layer lead to intrinsic non-uniformity in the devices. Previous studies have shown that this intrinsic non-uniformity can affect dynamic behaviors, ${ }^{2}$ modulation response, ${ }^{3}$ mode dynamics ${ }^{4}$ and selection, ${ }^{5}$ beam quality and optoelectric property, ${ }^{6}$ and threshold current, ${ }^{7}$ etc. Considerable modeling and simulation efforts ${ }^{2-8}$ are devoted to the understanding of the effects due to this particular type of intrinsic and time-dependent source of non-uniformity, as it is theoretically possible and practically desirable to individually identify different intrinsic and extrinsic contributions. Lising linear diffusion coefficient and gain model with rate equations, the authors in Ref. 2 found that both carrier diffusion and spontaneous emission suppress relaxation oscillation (RO) in gain-guided, smaller than about 10 micrometer lasers. Similarly, Wilt, et $\mathrm{al}^{3}$ concluded that lateral diffusion serves to damp the RO well, but would improve the modulation bandwidth poorly for narrow transverse stripe laser. More interestingly, as a control parameter, the diffusion coefficient was revealed ${ }^{4}$ to affect the stability of steady state lasing operation under many situations as it decreases. In addition, in the case of tapered amplifier, study ${ }^{6}$ shows that the far-field beam quality is greatly improved to a single lobe distribution with a large diffusion coefficient. The study indicates similar improvement for QW amplifiers as well. For vertical-cavity surface-emitting lasers (VCSELs), Dutta ${ }^{5}$ analyzed the effects of current spreading and carrier diffusion to transverse mode guiding, and found that current crowding at high density can help stabilize the fundamental mode operation. Shin, et al ${ }^{7}$ estimated diffusion-induced pumping loss as an overhead to the threshold current density for oxide-confined VCSELs. Finally, in contrast to other studies, Sarzala and .Nakwaski ${ }^{8}$ considered nonlinearity in the diffusion coefficient, and concluded that an average constant value of the coefficient is sufficient for reliable simulation results.

There are a few improvements foreseeable on these studies mentioned above, and the most apparent one is the application of a nonlinear gain model. To this end, we follow the so-called Effective Bloch-equations method, ${ }^{9}$

Further author information:

J.Z.L.: E-mail: jianzhng@nas.nasa.gov

S.H.C.: E-mail: cheung@nas.nasa.gov

C.Z.N.: E-mail: cning@nas.nasa.gov 
which actually goes further to take into account of the dynamics of the material polarization. However, what needs a reexamination is the study of the nonlinearity effect of the diffusion coefficient. In Ref. 8, Sarzala and Nakwaski used a generalized Einstein relation between the coefficient and carrier mobilities of electrons and holes, and empirical expressions for the mobilities to obtain the coefficient. What is questionable in this attempt is twofold. First, the expression used for the ambipolar diffusion coefficient is inappropriate in semiconductor lasers, as we will further discuss in Section 3. Furthermore, the empirical expressions for carrier mobilities are obtained for doped semiconductors, and should not be directly applied to semiconductor lasers. Detailed reasons are given in Section 2 and 3 . To remedy these shortcomings and obtain the mobilities and nonlinear coefficient microscopically, a hydrodynamic model ${ }^{10}$ has been developed by the present authors from first principles. It allows microscopic calculation of the lateral diffusion coefficient in the ambipolar regime. It turns out that the coefficient predicted by our model shows a positive carrier density dependence (cf Fig. 1, both solid and dashed curves), in contrast to Ref. 8. We mention that our results agree with other independent study. ${ }^{11}$ Thereby, together with a nonlinear gain model, we investigate the nonlinear effects of the diffusion coefficient on the operating and modulation characteristics of gain-guided, single-mode VCSELs. In this article, results will be presented for InGaAs/GaAs multiple QWs with a 7.5 micrometer current injection aperture in diameter and operating at 980 nanometers. Comparison will be made with selected cases of linear diffusion coefficient and conclusions will be drawn in terms of physical understandings.

This paper is organized as follows. In the next section, theoretical results will be summarized, and expressions for the nonlinear gain and density diffusion coefficient will be given. For completeness, we also describe the other field and interband polarization equations used for semiconductor lasers. Then, numerical results and discussions are presented, focusing on comparison of the linear cases and nonlinear case in our model. Finally, we will conclude the article with a summary and make some remarks for future directions.

\section{THEORY}

The hydrodynamic model, ${ }^{10,12}$ for carrier dynamics in the lateral active region of a semiconductor $\mathrm{QW}$ laser has been summarized in our previous work. ${ }^{10}$ Here we only list the relevant equations and briefly explain the involved physical quantities below. Before doing so, we mention that our model explicitly considers carrier-carrier scattering, which uniquely and conveniently leads to the ambipolar diffusion (AD) approximation in the limiting case of a dominant carrier-carrier scattering, as compared to carrier-LO phonon scattering. This is the case in which we have carried the current work, and we will discuss its plausibility. Furthermore, in this work we neglect the plasma heating effects, and their inclusion is beyond the scope of this article.

In the $\mathrm{AD}$ regime, charge neutrality eliminates the need for the Poisson equation and reduces the density equations for electrons and holes into a single one for the plasma density $N$, that is

$$
\partial_{t} N+\partial_{\vec{r}} \cdot \vec{J}_{N}=R_{N}
$$

where the density current $\vec{J}_{N}=-D_{a m} \partial_{\vec{r}} N$, and the ambipolar diffusion coefficient is obtained from both the diffusion coefficients and mobilities of electrons and holes in the electron-hole plasma in a semiconductor QW laser as follows,

$$
D_{a m}=\frac{\mu_{e} D_{h}+\mu_{h} D_{e}}{\mu_{e}+\mu_{h}}
$$

where $\mu_{e, h}$ and $D_{e, h}$ are the mobilities and diffusion coefficients of electrons and holes. We note that the above expression is the same as Eq. (51) in Ref. 11. We will comment on the inappropriate use of a similar formula (Eq. (1) in Ref. 8) in semiconductor lasers in Section 3.

To describe the semiconductor laser, we also need the macroscopic polarization and laser field equations, which are treated as in Ref. 9. The polarization is decomposed to two parts, electronic and background, as $P=P_{0}+P_{1}$, where the background contribution is given as $P_{0}=\epsilon_{0} \epsilon_{b} \chi_{0}\left(N, T_{l}\right) \mathcal{E}$, and the electronic part is dynamically described by

$$
\partial_{t} P_{1}=\left\{-\Gamma_{1}\left(N, T_{l}\right)+i\left[\omega_{c}-\omega_{1}\left(N, T_{l}\right)\right]\right\} P-i \epsilon_{0} \epsilon_{b} A_{1}\left(N, T_{l}\right) \mathcal{E},
$$

where the parameters $\chi_{0}$, the effective background susceptibility, $\Gamma_{1}$, the gain bandwidth, $\omega_{1}$, the peak gain frequency, and $A_{1}$, the Lorentzian oscillator strength, are fitted to microscopically computed values as a function of carrier 
density $N$ and lattice temperature $T_{l}{ }^{9}$ The laser field is described, after integrating over the assumed longitudinal mode profile, by

$$
\partial_{t} \mathcal{E}=\frac{i v_{g}}{2 K} \partial_{\vec{r}}^{2} \mathcal{E}+\frac{i v_{g} K \Gamma}{2 \epsilon_{g} \epsilon_{b}} P-\kappa \mathcal{E}
$$

Standard notations are used in the above equations, for example, $\omega_{c}$ is the central frequency, and $\Gamma$ is the optical mode confinement factor. Finally, the generation-recombination term in Eq. (1) is given as below,

$$
R_{N}=-\gamma_{N} N+\frac{\eta J}{e}-\frac{L_{m} \Gamma}{4 \hbar} \Im\left(P^{*} \mathcal{E}\right)
$$

where $J$ is the injection current with a spatial pumping profile and an quantum efficiency $\eta$, and $L_{m}$ is the aggregate active region width. This concludes the theoretical background section of the current work.

\section{SIMULATION RESULTS AND DISCUSSIONS}

Now we present and discuss our numerical simulation results based on the above equations, which have considered the nonlinearity in both the material gain and the diffusion coefficient in the limiting AD case. The simulations are run for a gain-guided MQW InGaAs/GaAs VCSEL operating at $\omega_{c}$ of $980 \mathrm{~nm}$. Injection current profile is uniformly circular with a diameter of $7.5 \mu \mathrm{m}$ in the middle and smoothly reduced to zero at $8.25 \mu \mathrm{m}$ following a $\cos ^{2}()$ behavior. The cavity length $L$ is $144 \mathrm{~nm}$ and $L_{m}$ is $36 \mathrm{~nm}$, which yields a $\Gamma$ of 0.25 . The other VCSEL parameters and simulation details can be found in Ning and Goorjian. ${ }^{13}$ Except the carrier lifetime $\left(1 / \gamma_{N}\right.$ or $\left.\tau_{n r}\right)$ of 0.25 nanosecond, other rates are computed microscopically and fitted as a function of $N$ and $T_{l}$. Furthermore, we use an injection current $J$ of $1.783 \mathrm{KA} / \mathrm{cm}^{2}$, which is about $40 \%$ above the threshold current for the case of a constant diffusion coefficient of $20 \mathrm{~cm}^{2} / \mathrm{s}$. And the lattice temperature $T_{l}$, as well as the plasma temperature $T_{p}$, is assumed to be a constant of $295 \mathrm{~K}$ throughout our simulations. Finally, we use finite difference method to solve the time-dependent equations in the QW plane.

First we show the comparison of different ambipolar diffusion coefficients in an 8 nanometer GaAs quantum well as a function of the plasma density in Fig. 1. As we mention at the beginning of Section 2 , the coefficient we use in the current study for InGaAs MQW lasers, similar to the solid curve in the figure, is calculated in the limiting case where carrier-carrier scattering is the dominant mechanism that determines the carrier transport. This is true when the plasma density is high enough as in the lasing region. Also shown in the figure is the microscopic result for the realistic case (dashed curve) which differs from the limiting case at the low end of the plasma density, where carrier-LO phonon scattering becomes the mechanism that dictates carrier transport and thus flattens the AD coefficient since the scattering is not density-dependent at low plasma density. The main reason we use the limiting case is following. In the case of single mode operation for a gain-guided VCSEL, the field distribution is restricted around the middle of the pumping zone in a relatively smaller region than the spreading of the plasma density (cf Fig. 2). The important region where the nonlinearity in the coefficient takes effect on the laser field is not restricted to where the field distribution is appreciable, but actually covers the whole active region. And the reason is the self-adaptive nature of nonlinear processes. In essence, the nonlinear dependence of the coefficient on the plasma density averages out such that an effective coefficient can be defined. Due to this self-adaptive nature, which is still true even after the plasma density is coupled to the laser field, the corresponding density to this effective coefficient is at half height of the density distribution, roughly speaking. Our previous simulation results ${ }^{10}$ actually show that the plasma density spreads to a region with a full width at half height of about the laser field distribution (cf Fig. 2 as well).This self-adaptiveness of the nonlinear coefficient not only justifies our selection of the coefficient, but also offers us the explanation for our main conclusion of this study, that is, the effects of nonlinearity in the diffusion coefficient seemingly follow the behaviors as if predicted by an effective constant diffusion coefficient, with the corresponding density approximately at half height of the plasma density distribution. This is the same conclusion as in Ref. 8, despite the fact that that work treated the AD coefficient questionably as we discuss now. To make a comparison, the coefficient used in that work is plotted as the dotted curve in Fig. 1. There are mainly two comments we want to address about that work. First of all, it adopted the following expression to obtain the AD coefficient,

$$
D_{a m}=\frac{n+p}{\frac{n}{D_{h}}+\frac{p}{D_{e}}}
$$




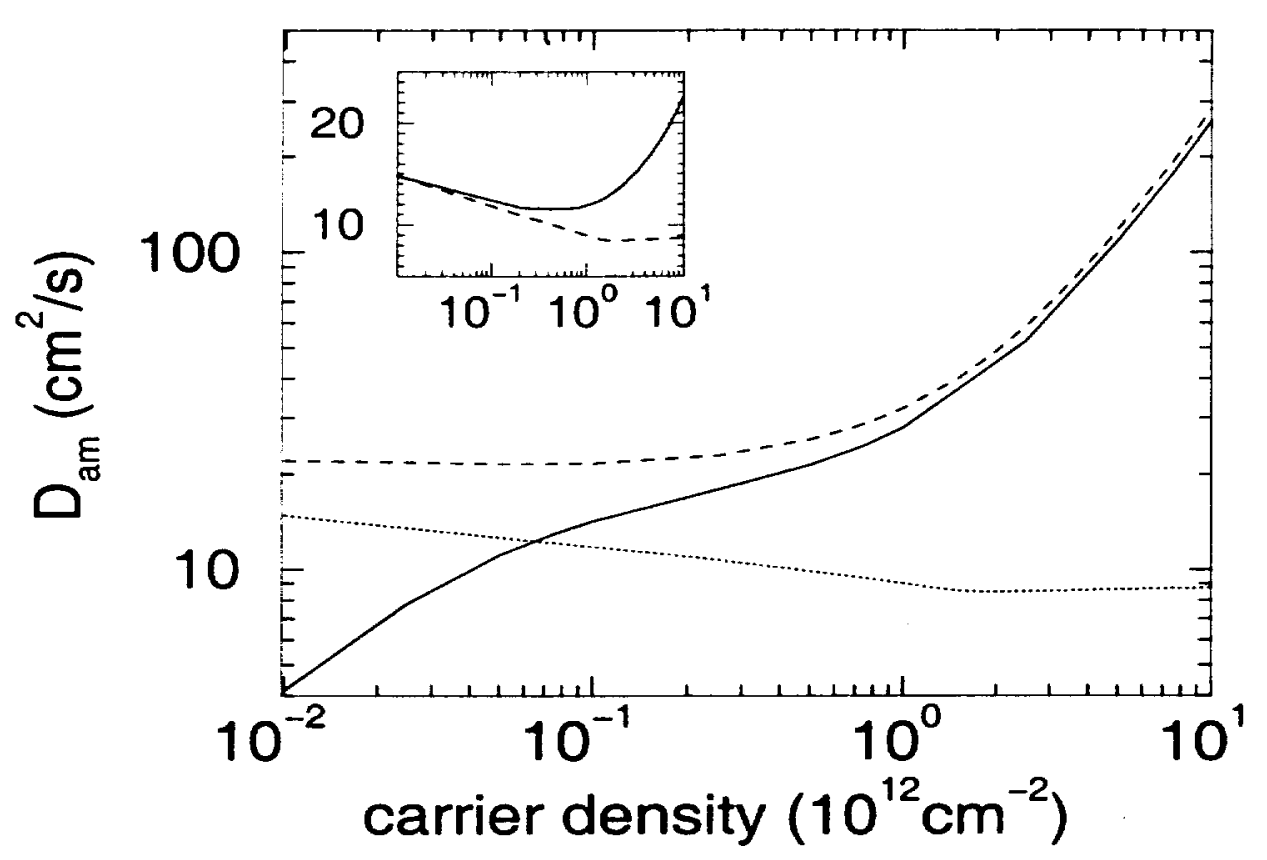

Figure 1. Ambipolar diffusion coefficients vs. plasma density for an 8 nanometer GaAs quantum well. Solid curve is the limiting AD case as used in this study for InGaAs, the dashed curve is the realistic AD case which takes into full account of the carrier-LO phonon scattering at low density, and the dotted curve is used in Ref. 8. The former two curves are theoretical data obtained from microscopic calculations, while the latter is obtained empirically. The inset shows the comparison of the results obtained using Eq. (2) in this article and Eq. (1) in Ref. 8 from the same empirical data in Ref. 8.

where $n$ and $p$ are the concentrations of electrons and holes, respectively, and $n=p$ in the ambipolar regime. We note that the above expression is equivalent to Eq. (2) when the Einstein relation is valid. Eq. (6) gives different AD coefficient from that by Eq. (2), as exhibited in the inset of Fig. 1. The solid curve is obtained from Eq. (2), while the dashed curve is acquired with Eq. (6) for $n=p$. Clearly the difference lies in the high density side, and the density dependence of the coefficient is the opposite around the transparency density. The reason is that the above expression fails when the carriers become statistically degenerate at high enough density, even though both equations agree at low density where Maxwell distribution applies for electrons and holes. As mentioned in that work, a generalized Einstein relation needs to be used between the diffusion coefficient and the mobility of either electrons or holes when they become degenerate, and correspondingly a density-dependent correction factor is introduced. ${ }^{14}$ However, what is important here is that both curves depend quite weakly on the density in the lower density regime. This key feature is why the study in Ref. 8 leads to the conclusion, which is the same as ours by coincidence. As further illustrated in the main panel of the figure, the coefficient used in that work merely varies by a factor of two over three orders of magnitude change in the density such that replacement of the weakly density-dependent coefficient with an averaged one is not very conclusive. The second comment concerns with the adoption of empirical mobility expressions valid for doped semiconductors to intrinsic electron-hole plasmas in semiconductor lasers. Because of different scattering mechanisms, especially carrier-carrier scattering at high density for electron-hole plasmas and carrier-ionized impurity scattering at high density for doped semiconductors, at work that influence the transport properties, including carrier mobilities, that is why our coefficients can be more than an order of magnitude larger than in Ref. 8. Certainly, we would not rule out other contributions in doped semiconductors to this discrepancy.

Fig. 2 shows the cross sections of fundamental mode and density distribution through the circular center of the gain-guided VCSEL and compares the case of a nonlinear AD coefficient, curve (a), to three representative linear cases, that is (1) curve (b)-dotted line, with a constant coefficient of $54.115 \mathrm{~cm}^{2} / \mathrm{s}$, which corresponds to a plasma density of $2.5 \times 10^{12} \mathrm{~cm}^{-2}$; (2) curve (c)-dashed line, with a constant coefficient of $23.838 \mathrm{~cm}^{2} / \mathrm{s}$, which corresponds to a plasma density of $7 \times 10^{11} \mathrm{~cm}^{-2}$; (3) curve (d)-long dashed line, with a constant coefficient of $7.5 \mathrm{~cm}^{2} / \mathrm{s}$, which corresponds to a plasma density of $2.5 \times 10^{10} \mathrm{~cm}^{-2}$. This choice of the linear cases allows us more convincingly 


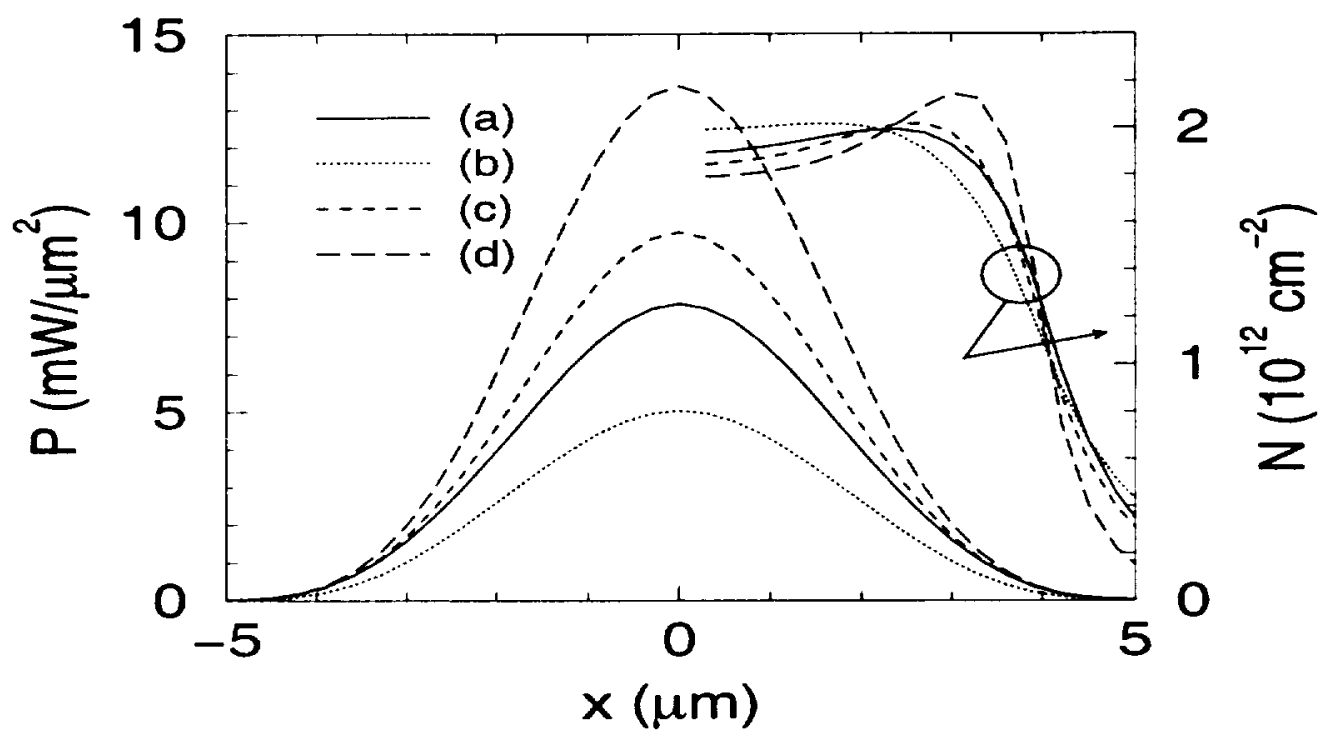

Figure 2. Fundamental mode and density distribution cross section through the circular center of the InGaAs VCSEL. Solid curve (a) is the fully nonlinear AD coefficient case, in comparison with the constant coefficient cases (b) $D_{a m}=54.115$-dotted line; (c) $D_{a m}=23.838$-dashed line; (d) $D_{a m}=7.5$-long dashed line. All units are $\mathrm{cm}^{2} / \mathrm{s}$. Only half of the density distribution is shown since it is circularly symmetric.

reaching the main conclusion of this study with the help of working in the limiting AD case, in contrast with the realistic $A D$ case, since the latter doesn't allow much change in the coefficient at the lower density regime. This is the minor reason we have chosen to work in the limiting $\mathrm{AD}$ case. In other words, in the realistic $\mathrm{AD}$ case the coefficient varies as little as two-fold as the density is lower than $10^{12} \mathrm{~cm}^{-2}$. As seen in the figure, the nonlinear case has a value between that of case (b) and (c), or roughly speaking in terms of linear interpolation, corresponds to an effective constant coefficient of $\sim 30 \mathrm{~cm}^{2} / \mathrm{s}$, which is the value at the density of $\sim 10^{12} \mathrm{~cm}^{-2}$ according to our limiting AD case-solid curve in Fig. 1. That is to say that the effective coefficient comes about at the density of the half height of the distribution. Another feature that easily catches attention is the increase in laser intensity as the diffusion coefficient decreases, from case (b) to (d). And correspondingly, the spatial hole burning increases in the density distribution. It is fairly easy to see why increase in the coefficient results in reduction in laser intensity, and the reason is because less population inversion is achieved as diffusion increases. Overall, the mode is more confined than the density distribution simply because of the gain guiding effect and the absorption by the material when the plasma density is below the transparent point. The consequences of this feature have been seen in the earlier discussion, thus will not be repeated here.

Figure 3 shows a comparison of the average near-field power density as a function of the injection current density for the four cases: nonlinear case (a) and three linear cases (b-d). There are two features which are consistent with what we have shown and discussed thus far. Specifically, it is obvious that the threshold injection current for the VCSEL increases with the coefficient. The explanation for this phenomenon is straightforward. Because of carrier diffusion, and thus current spreading, less efficiently the current density can be utilized for population inversion, which leads to the increase of threshold current with diffusion coefficient. An additional feature has to do with the nonlinear case. As in Fig. 2, it sits in between case (b) and (c). It is estimated by linear interpolation that the LI curve for the nonlinear case corresponds to an effective constant coefficient of $\sim 30 \mathrm{~cm}^{2} / \mathrm{s}$, as in the earlier analysis for simulation result in Fig. 2. This is another demonstration which allows us to come to the main conclusion of this study, as stated above and will be reiterated in Section 4.

The final simulation result is for small-signal modulation bandwidth for the same four cases as detailed above. The amplitude of the current modulation is $5 \%$ of the DC injection current density. The simulation result is produced by fitting the linear response of the VCSEL to the modulation pattern of the current density at various frequencies. First, we note that the modulation resonance occurs near $20 \mathrm{GHz}$, which is larger than realistic devices because we have used a smaller carrier lifetime $r_{n r}$ of 0.25 nanosecond. Second, consistent with the earlier results in this study, 


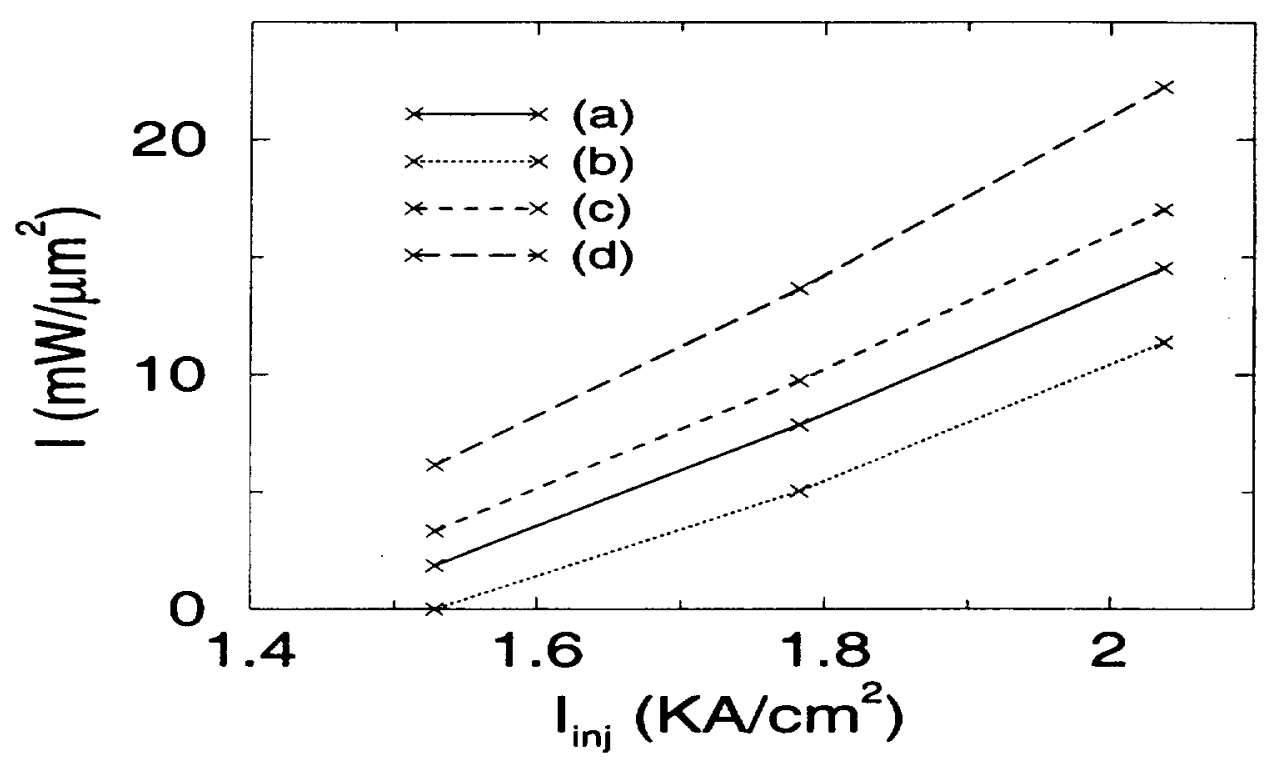

Figure 3. Average near-field power density vs. injection current density. The different cases follow the same line types as used in Figure 2 and throughout this article.

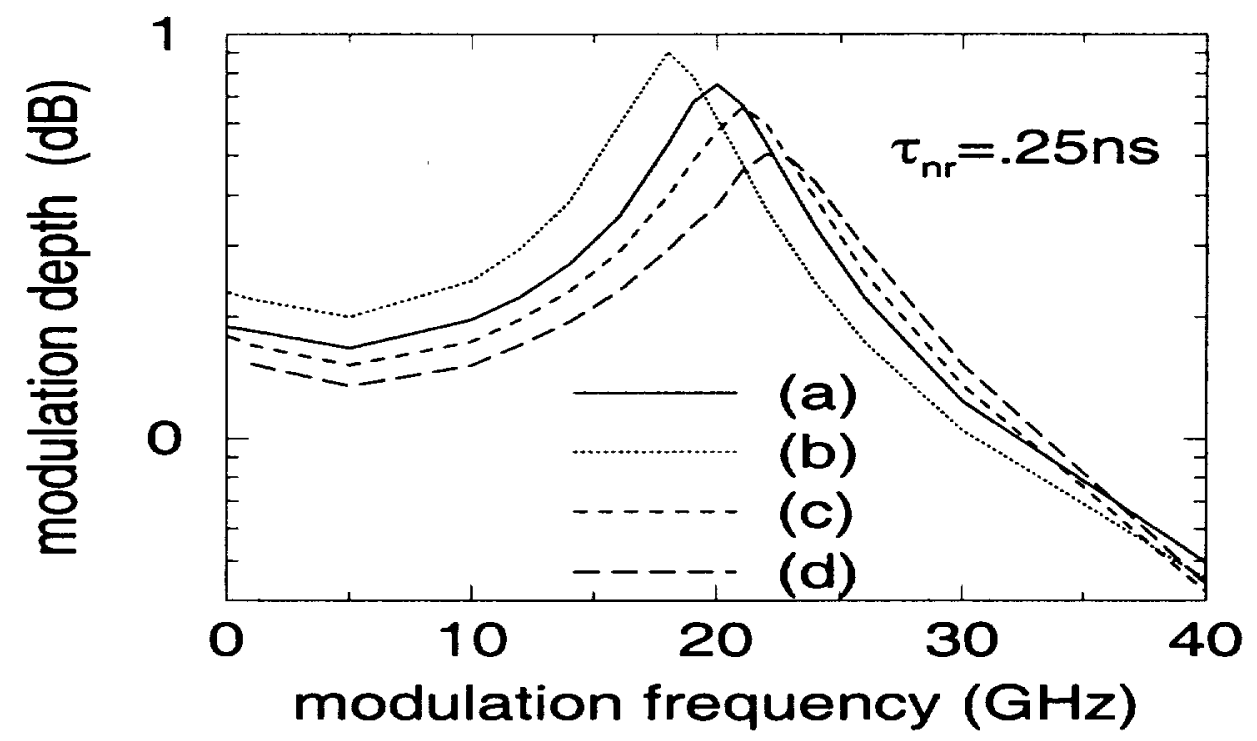

Figure 4. Small-signal modulation bandwidth of the VCSEL in different cases. Same line types as in Figure 2 and elsewhere in this article are used.

the increase in the coefficient leads to narrower modulation bandwidth $f_{3 d B}$, which can be understood qualitatively as due to increase in the threshold current density, that is to say an equivalent deduction in the pumping current density as we have fixed the pumping current density to about $40 \%$ above the threshold level. It is known that increase in pumping level drives the material differential gain up, which in turn raise the response speed of the semiconductor laser. It is also possible that dynamic enhancement is resulted from the decrease in the coefficient. In our future work, we will perform a quantitative analysis of this possibility to clearly identify how much of the bandwidth increase is a result of decrease in the threshold pumping current for smaller diffusion coefficient, thus an effective increase of the DC pumping level. Third, as noted, the nonlinear case (a) falls between case (b) and (c). Similarly the same estimate can be carrier out to find an effective diffusion coefficient, and similar result is achieved as above. Thus, the modulation result further validates our main conclusion. 
To conclude this section, we mention that the fundamental reason for the validity of an effective constant diffusion coefficient in replacement of the nonlinear one is because of the self-adaptive nature of the nonlinear diffusive process. This also explains why the same conclusion is reached by this study and Ref. 8, despite of obvious differences.

\section{SUMMARY}

Carrier diffusions in the plane of semiconductor quantum wells play an important part in the static operations and dynamic responses of semiconductor lasers, as further corroborated in the current study. In this article, we have applied a newly developed hydrodynamic model by the present authors from the semiconductor Bloch equations to investigate the nonlinear effects of the diffusion, as measured by the so-called ambipolar diffusion coefficient. The model allows us to microscopically calculate carrier diffusion coefficients, which are nonlinear functions of the carrier density and plasma temperature. We first look into earlier nonlinearity study by other researchers and make comparison and comments about both approaches. It is found that in spite of the great differences between the two approached, such as in the ambipolar diffusion coefficients used (cf Fig. 1), but owing to the self-adaptive nature of the nonlinear effects, an effective constant diffusion coefficient can be equivalently used to predict both $\mathrm{AC}$ and DC performances of the semiconductor QW laser. This coefficient corresponds to a density at about half height of its spreaded distribution. This conclusion is supported by simulation results for near-field intensity and plasma density distributions, for laser output power as a function of pumping current level, for the small-signal modulation bandwidth. The simulations are designed to have the nonlinear diffusion case compared with three representative linear cases such that a simple quantitative estimation can be made for the nonlinearity effects. Finally, the results also show the disadvantages for a larger diffusion coefficient, which will increases the threshold current, reduce the laser output power, and decrease the modulation speed of the semiconductor laser.

\section{FUTURE DIRECTIONS}

Before we conclude this article, we would like to discuss some future directions as a continuation of our current study. Obviously, in a gain-guided device, the density distribution changes less drastically than in a index-guided case, as current spreading is less restrictive. Thus, the nonlinearity effects could be enhanced in a more restricted geometry for the device because more likely a greater density gradient can be achieved. Also, as injection level increases, the variation in the density distribution in a restrictive device design may exhibit pronounced difference from its gainguided counterpart as density moves to higher regime, thus possible stronger nonlinear effects as indicated in Fig. 1. Furthermore, combination of all these considerations for VCSEL arrays could bring new scenes into perspective due to increase in the plasma density and its gradient. Finally, future study will also aim at unambiguous identification of the static and dynamic consequences of the nonlinearity in the coefficient by separating the AC and DC effects through well-designed simulations.

\section{ACKNOWLEDGMENTS}

This work is supported by the IPT program at NASA Ames Research Center.

\section{REFERENCES}

1. I. Vurgaftman and J. R. Meyer, "Effects of bandgap, lifetime, and other nonuniformities on diode laser thresholds and slope efficiencies," IEEE J. Select. Topics Quantum Electron. 3, pp. 475-484, 1997.

2. N. Chinone, K. Aiki, M. Nakamura, and R. Ito, "Effects of lateral mode and carrier density profile on dynamic behaviors of semiconductor lasers," IEEE J. Quantum Electron. QE-14, pp. 625-631, 1978.

3. D. Wilt, K. Y. Lau, and A. Yariv, "The effect of lateral carrier diffusion on the modulation response of a semiconductor laser," J. Appl. Phys. 52, pp. 4970-4974, 1981.

4. P. A. Khandokhin, I. V. Koryukin, Y. I. Khanin, and P. Mandel, "Influence of carrier diffusion on the dynamics of a two-mode laser," IEEE J. Quantum Electron. 31, pp. 647-652, 1995.

5. N. K. Dutta, "Analysis of current spreading, carrier diffusion, and transverse mode guiding in surface emitting lasers," J. Appl. Phys. 68, pp. 1961-1963, 1990.

6. J.-W. Lai and C.-F. Lin, "Carrier diffusion effect in tapered semiconductor-laser amplifier," IEEE J. Quantum Electron. 34, pp. 1247-1256, 1998. 
7. J. H. Shin, H. E. Shin, and Y. H. Lee, "Effect of carrier diffusion on oxidized vertical-cavity surface-emitting lasers determined from lateral spontaneous emission," Appl. Phys. Lett. 70, pp. 2652-2654, 1997.

8. R. P. Sarzala and W. Nakwaski, "Carrier diffusion inside active regions of gain-guided vertical-cavity surfaceemitting lasers," IEE Proc.-Optoelectron. 144, pp. 421-425, 1997.

9. C. Z. Ning, R. A. Indik, and J. V. Moloney, "Effective Bloch-equations for semiconductor lasers and amplifiers," IEEE J. Quan. Electron. 33, pp. 1543-1550, 1997.

10. J. $\mathrm{Li}$ and $\mathrm{C}$. Z. Ning, "Theory and simulation of self- and mutual-diffusions of carrier density and temperature in semiconductor lasers," in Physics and Simulation of Optoelectronic Devices IX, Y. Arakawa, P. Blood, and M. Osinski, eds., SPIE Proc. 4283, pp. 267-274, 2001.

11. O. Hess and T. Kuhn, "Maxwell-Bloch equations for spatially inhomogeneous semiconductor lasers. I. theoretical formulation," Phys. Rev. A 54, pp. 3347-3359, 1996.

12. J. Li, S. H. Cheung, and C. Z. Ning, "A hydrodynamic approach to spatially inhomogeneous semiconductor lasers." unpublished.

13. C. Z. Ning and P. M. Goorjian, "Microscopic modeling and simulation of transverse-mode dynamics of verticalcavity-surface emitting lasers," J. Opt. Soc. Am. B 16, pp. 2072-2082, 1999.

14. P. T. Landsberg, "The Einstein relation," Proc. IEEE 61, p. 476, 1973. 